\title{
Can Pap smear and colposcopy predict cervical pathology prior to hysterectomy?
}

\author{
Original \\ Shimaa Mostafa Abd-El-Fatah, Mohamed El Sharkawy, Nawara Mohamed Hashish, \\ Article \\ Ahmed Saeed, Ahmed Alaa-El-Din Wali \\ Department of Obstetrics and Gynecology, Faculty of Medicine, Cairo University, \\ Cairo, Egypt
}

\begin{abstract}
Aim: To evaluate the accuracy of Pap smear and colposcopy as predictors of cervical histopathology in patients undergoing total hysterectomy for benign indications, and to determine the incidence of unexpected cervical pathology in these patients.

Study Design: Prospective observational study

Patients and Methods: One hundred women scheduled for total hysterectomy for benign indications were subjected to preoperative Pap smear and colposcopy, at Kasr Al-Ainy Hospital, Cairo University, Egypt, during the period from September 2017 to March 2018. Findings were compared with the histopathological results of the cervices of the hysterectomy specimens.

Results: Out of 100 patients included in our study, 13 had an abnormal Pap smear and 44 had abnormal findings on colposcopy. Abnormal cervical pathology was found in 30 hysterectomy specimens. Pap smear had a sensitivity and specificity of $33.3 \%$ and $95.7 \%$, respectively, while colposcopy had a sensitivity and specificity of $96.7 \%$ and $78.6 \%$, respectively. The incidence of unexpected cervical pathology was $66.7 \%$ depending on the Pap alone, and $3.3 \%$ when depending on colposcopy.

Conclusion: Pap smear has a good specificity, but a low sensitivity in predicting cervical histopathology. Meanwhile, colposcopy has a high sensitivity and a reasonable specificity in predicting the histopathology. Colposcopy has a higher sensitivity and specificity as a pathology predictor, when compared to Pap smear.
\end{abstract}

Key Words: Cancer cervix, cervical screening, CIN, colposcopy, cytology, histopathology, hysterectomy, Pap smear

Received: 15 October 2019, Accepted: 15 December 2019

Corresponding Author: Ahmed Alaa-El-Din Wali, Department of Obstetrics and Gynecology, Faculty of Medicine, Cairo University, Cairo, Egypt, Tel.: +201001735088, E-mail: ahmed.wali@kasralainy.edu.eg

ISSN: 2090-7265, February 2020, Vol. 10, No. 1

\section{INTRODUCTION}

Hysterectomy is the most commonly performed major gynecological operation worldwide. It can be done for both malignant and non-malignant gynecological conditions $^{[1,2]}$.

Cancer cervix is the third most common gynecological malignancy - after breast and endometrial cancer - which can affect women. Invasive cancer cervix is a preventable disease as it remains in a pre-invasive stage for long periods, and available screening programs allow early detection and management of cervical dysplasia ${ }^{[3,4]}$. Cytology (Pap smear) is a simple, non-invasive and effective method for detection of pre-malignant changes in the cervix and vagina. Other available tools are colposcopy and histopathology. The colposcope allows direct visualization of the cervix enabling a view of the transformation $z e^{[5,6]}$. Incidental pathological findings have been found in cervical tissues in hysterectomy specimens done for benign indications; hence, increasing the importance of screening females 35 to 65 years by Pap smear and colposcopy ${ }^{[7]}$.

In Egypt -where there is no official cervical screening program - preoperative cytology and colposcopy allow modification of the surgical plan, thus avoiding further surgeries and complications due to metastasis or stump recurrence ${ }^{[8]}$.

The aim of our work was to evaluate the accuracy of preoperative assessment of the cervix by colposcopy and Pap smear, compared to histopathological examination of the cervix after total hysterectomy.

\section{MATERIALS AND METHODS}

A prospective study was conducted among one hundred women who presented with various gynecological complaints at the gynecology outpatient clinic in Kasr 
Al-Ainy Hospital, Cairo University, Egypt, during the period from September 2017 to March 2018. The Ethics Committee of Cairo University approved the study protocol. Written informed consent was obtained from all patients who met the inclusion criteria after the nature of the procedures were fully explained. Patients who were admitted for total hysterectomy for benign indications were told that they would be subjected to preoperative Pap smear and colposcopy, then the results would be compared to the histopathological findings of the cervices of the hysterectomy specimens. Inclusion criteria were: married or previously married women of age group 35 to 70 years undergoing total hysterectomy. Exclusion criteria were: virgins, age above 70 years and below 35 years and patients who already had total hysterectomy, patients already diagnosed or treated for cervical cancer.

All participants were subjected to full history taking, general and abdominal examination, and local examination (including per vaginal, bimanual and speculum examination for inspection of the cervix). Conventional Pap smear was carried out using Ayres spatula to scrape the cervix in 360 degrees. The material collected was spread on a glass slide which was immersed in alcohol $95 \%$ as a fixative for at least 20 minutes then stained by Papanicolaou's stain and examined under a light microscope. The cytological interpretation of the smears was done according to the Bethesda system 2014 ${ }^{[9]}$ : Negative for Intraepithelial Lesion or Malignancy (NILM), Low-grade Squamous Intraepithelial Lesion (LSIL), High-grade Squamous Intraepithelial Lesion (HSIL) and Malignancy. A normal smear was defined as one showing NILM, while an abnormal smear was defined as a smear with LSIL, HSIL or Malignancy. Colposcopic examination was done by a video colposcope "VICO", Karl Kaps GmbH and Co.KG, Asslar/Wetzlar, Germany. The cervix was washed with saline to remove any excess secretions. The green filter was applied to detect abnormal vessels. Acetic acid 3\% was applied to the cervix using cotton swabs to enhance definition of the squamo-columnar junction and transformation zone. Schiller's Iodine test was done by applying Lugol's iodine to the cervix, which stains mature squamous epithelial cells in a mahogany color due to the high cellular glycogen content. The areas with no iodine uptake especially if preceded by aceto-white areas were considered abnormal and biopsied. Squamo-columnar junction and lesions of the cervix were evaluated by colposcopy as areas of aceto-white changes, areas of punctuation, areas of mosaicism and areas of abnormal blood vessels. A colposcopic examination showing any of the forementioned lesions was defined as abnormal.

After total hysterectomy, specimens were fixed in buffered formalin $10 \%$, and sent to the histopathology laboratory to be embedded in paraffin blocks. From these paraffin blocks slides were prepared for staining with hematoxylin and eosin for histopathological examination. Results were interpreted as: cervicitis, CIN1 (cervical intraepithelial neoplasia), CIN2, CIN3 and SCC (squamous cell carcinoma). Abnormal histopathology was defined as a one showing CIN or SCC.

\section{RESULTS}

The total number of women invited to participate in the study was 129 women. 21 were excluded for not meeting the inclusion criteria. Two women withdrew from the study, another two had unsatisfactory pap smears and four had unsatisfactory colposcopy. Finally, one hundred women participated in the study. The age of studied group ranged from 40 to 58 years with a mean and standard deviation (SD) of $47.79 \pm 4.25$ years. The duration of marriage was between 6 and 38 years with mean and SD of $23.13 \pm 8.06$ years (Table 1).

Most of the patients $(88 \%)$ were married at the time of the study. Those married once in their lifetime were $96 \%$, who used contraception were $72 \%$, non-smokers were $97 \%$, and multipara more than 4 times were $41 \%$ of the participants. The most common complaint was menstrual disorder constituting $40 \%$ of all complaints (Table 2).

When correlating the patients' complaints to the final histopathology (of the cervices in the hysterectomy specimens), patients in whom menstrual disorder was the main complaint, CIN was found in 13 out of the 40 patients $(32.5 \%)$. CIN was also found in 9 of the 32 abdominal pain patients $(28 \%)$, and in 6 of the 25 post-menopausal bleeding patients $(24 \%)$. SCC was seen in only one patient in the study, whose complaint was menstrual disorder (Table 3).

Table 1: Age and duration of marriage as groups

\begin{tabular}{lccc}
\hline & Groups & Frequency & Percent \\
\hline \multirow{3}{*}{ Age (years) } & $40-45$ & 26 & $26 \%$ \\
& $45-50$ & 48 & $48 \%$ \\
& $>50$ & 26 & $26 \%$ \\
Duration of marriage (years) & $5-10$ years & 15 & $15 \%$ \\
& $10-20$ years & 28 & $28 \%$ \\
\hline
\end{tabular}


Table 2: Demographic data of the patients

\begin{tabular}{|c|c|c|c|}
\hline & & Frequency & Percent \\
\hline \multirow{3}{*}{ Marital status } & Married & 88 & $88 \%$ \\
\hline & Widow & 9 & $9 \%$ \\
\hline & Divorced & 3 & $3 \%$ \\
\hline \multirow{3}{*}{ Number of ma } & Once & 96 & $96 \%$ \\
\hline & & & \\
\hline & Twice & 4 & $4 \%$ \\
\hline \multirow{3}{*}{ Contraception } & Yes & 72 & $72 \%$ \\
\hline & & & \\
\hline & No & 28 & $28 \%$ \\
\hline \multirow{2}{*}{ Smoking } & Yes & 3 & $3 \%$ \\
\hline & & & 070 \\
\hline & & & \\
\hline \multirow{5}{*}{ Parity } & Nullipara & 2 & $2 \%$ \\
\hline & Para 1 & 12 & $12 \%$ \\
\hline & Para 2 & 21 & $21 \%$ \\
\hline & Para 3 & 24 & $24 \%$ \\
\hline & Para 4 or more & 41 & $41 \%$ \\
\hline \multirow{5}{*}{ Complaint } & Menstrual disorder & 40 & $40 \%$ \\
\hline & Post-menopausal bleeding & 25 & $25 \%$ \\
\hline & & & \\
\hline & Lower abdominal pain & 32 & $32 \%$ \\
\hline & Uterine descent & 3 & $3 \%$ \\
\hline
\end{tabular}

Table 3: Complaint and histopathology

\begin{tabular}{|c|c|c|c|c|c|c|c|}
\hline \multirow{6}{*}{ Complaint } & & \multicolumn{5}{|c|}{ Histopathology findings } & \multirow{2}{*}{ Total } \\
\hline & & Cervicitis & CIN1 & CIN2 & CIN3 & $\mathrm{SCC}$ & \\
\hline & Menstrual disorder & $26 \%$ & $10 \%$ & $3 \%$ & - & $1 \%$ & $40 \%$ \\
\hline & Postmenopausal bleeding & $19 \%$ & $5 \%$ & $1 \%$ & - & - & $25 \%$ \\
\hline & Abdominal pain & $23 \%$ & $6 \%$ & $2 \%$ & $1 \%$ & - & $32 \%$ \\
\hline & Uterine descent & $2 \%$ & $1 \%$ & - & - & - & $3 \%$ \\
\hline Total & & $70 \%$ & $22 \%$ & $6 \%$ & $1 \%$ & $1 \%$ & $100 \%$ \\
\hline
\end{tabular}


Regarding the clinical appearance of cervix by speculum examination, we had three main findings, namely normal cervix, cervical erosion and suspicious cervix. Cervical erosion was the commonest abnormal finding (84\%). On correlating with the histopathological findings, 18 of the 84 patients with erosions had CIN (21\%). Two patients had a suspicious cervix, one was found to have CIN 2 by histopathology and the other had SCC (Table 4).
Relation between age, parity and duration of marriage, and the histopathological findings are shown in Tables 5-7. Highest frequency of CIN was found in the age group of 45 to 50 years (Tables 5). The higher the patients' parity, the higher the frequency of CIN (Tables 6). Also, the longer the duration of marriage the higher the frequency of CIN (Tables 7).

Table 4: Cervical examination and histopathology

\begin{tabular}{|c|c|c|c|c|c|c|c|}
\hline \multirow{5}{*}{ Cervical examination } & & \multicolumn{5}{|c|}{ Histopathology findings } & \multirow{2}{*}{ Total } \\
\hline & & Cervicitis & CIN1 & CIN2 & CIN3 & $\mathrm{SCC}$ & \\
\hline & Normal & $4 \%$ & $10 \%$ & - & - & - & $14.0 \%$ \\
\hline & Erosion & $66 \%$ & $12 \%$ & $5 \%$ & $1 \%$ & - & $84.0 \%$ \\
\hline & Suspicious & - & - & $1 \%$ & - & $1 \%$ & $2.0 \%$ \\
\hline Total & & $70 \%$ & $22 \%$ & $6 \%$ & $1 \%$ & $1 \%$ & $100.0 \%$ \\
\hline
\end{tabular}

Table 5: Age and histopathology

\begin{tabular}{lccccccc}
\hline & & \multicolumn{5}{c}{ Histopathology findings } \\
\cline { 3 - 6 } Age group (years) & & Cervicitis & CIN1 & CIN2 & CIN3 & SCC & Total \\
\cline { 2 - 6 } & $40-45$ & $17 \%$ & $8 \%$ & $1 \%$ & - & - & $26.0 \%$ \\
& $45-50$ & $33 \%$ & $10 \%$ & $4 \%$ & $1 \%$ & - & $48.0 \%$ \\
& $>50$ & $20 \%$ & $4 \%$ & $1 \%$ & - & $1 \%$ & $26.0 \%$ \\
Total & & $70 \%$ & $22 \%$ & $6 \%$ & $1 \%$ & $1 \%$ & $100.0 \%$ \\
\hline
\end{tabular}

Table 6: Parity and histopathology

\begin{tabular}{|c|c|c|c|c|c|c|c|}
\hline \multirow{7}{*}{ Parity } & & \multicolumn{5}{|c|}{ Histopathology findings } & \multirow{2}{*}{ Total } \\
\hline & & Cervicitis & CIN1 & CIN2 & CIN3 & $\mathrm{SCC}$ & \\
\hline & Nullipara & $1 \%$ & $1 \%$ & - & - & - & $2.0 \%$ \\
\hline & Para 1 & $10 \%$ & $2 \%$ & - & - & - & $12.0 \%$ \\
\hline & Para 2 & $16 \%$ & $4 \%$ & $1 \%$ & - & - & $21.0 \%$ \\
\hline & Para 3 & $19 \%$ & $3 \%$ & $2 \%$ & - & - & $24.0 \%$ \\
\hline & Para 4 or more & $24 \%$ & $12 \%$ & $3 \%$ & $1 \%$ & $1 \%$ & $41.0 \%$ \\
\hline Total & & $70 \%$ & $22 \%$ & $6 \%$ & $1 \%$ & $1 \%$ & $100.0 \%$ \\
\hline
\end{tabular}


Table 7: Duration of marriage and histopathology

\begin{tabular}{|c|c|c|c|c|c|c|c|}
\hline \multirow{5}{*}{ Duration of marriage (years) } & & \multicolumn{5}{|c|}{ Histopathology findings } & \multirow{2}{*}{ Total } \\
\hline & & Cervicitis & CIN1 & CIN2 & CIN3 & SCC & \\
\hline & $5-10$ & $12 \%$ & $2 \%$ & $1 \%$ & - & - & $15 \%$ \\
\hline & $10-20$ & $18 \%$ & $8 \%$ & $2 \%$ & - & - & $28 \%$ \\
\hline & $>20$ & $40 \%$ & $12 \%$ & $3 \%$ & $1 \%$ & $1 \%$ & $57 \%$ \\
\hline Total & & $70 \%$ & $22 \%$ & $6 \%$ & $1 \%$ & $1 \%$ & $100 \%$ \\
\hline
\end{tabular}

Comparison of Pap smear with histopathology showed that out of total 13 patients with abnormal Pap smear, 10 (77\%) had abnormal histopathology, and of the 87 patients with normal smears, 67 (77\%) had normal histopathology (Table 8$)$. There were 3 false positive cases $(23 \%)$. There were 20 false negative cases i.e. cytology missed 20 cases with abnormal histopathology. The calculated sensitivity and specificity for Pap smear were 33.3\% and 95.7\% compared to histopathology, denoting a very low sensitivity compared to its specificity. Colposcopy findings were normal in 56 (56\%) women, and 44 (44\%) had abnormal findings. Among the 44 with abnormal colposcopy, 29 of the $44(66 \%)$ had abnormal histopathology, while in the remaining $15(34 \%)$ women, the histopathology was normal (Table 9). On the other hand, out of the 56 women with normal colposcopy, histopathology was abnormal in one case i.e. $1.8 \%$ false negative. The calculated sensitivity and specificity of colposcopy findings compared to histopathology was $96.7 \%$ and $78.6 \%$, respectively.

A correlation of Pap smear findings to colposcopy findings is demonstrated in Table 10. All 13 women with abnormal smears had an abnormal colposcopy examination. On the other hand, of the 87 women with normal smears, $31(35.6 \%)$ had abnormal colposcopy. The sensitivity and specificity of Pap smear was 29.5\% and $100 \%$, respectively, compared to colposcopy. The total number of women who had an abnormal cervical histopathology - CIN or malignancy - in their hysterectomy specimens was 30 cases. Pap smear could predict only ten of them, while colposcopy missed only one case. However, colposcopy had a false positive result in 14 cases with a normal histopathology, meanwhile Pap smear had only three false positive results. This means that in our present finding, the incidence of unexpected cervical pathology was $66.7 \%$ depending on the Pap alone, and only $3.3 \%$ when depending on colposcopy.

Table 8: Correlation of Pap smear to histopathology

\begin{tabular}{lcccccc}
\hline \multirow{2}{*}{ Pap smear } & \multicolumn{5}{c}{ Histopathology findings } & Total \\
\cline { 2 - 5 } NILM & Cervicitis & CIN1 & CIN2 & CIN3 & SCC & $87 \%$ \\
LSIL & $67 \%$ & $19 \%$ & $1 \%$ & - & - & - \\
HSIL & $3 \%$ & $3 \%$ & $2 \%$ & - & - & $8 \%$ \\
Malignancy & - & - & $3 \%$ & $1 \%$ & - & $4 \%$ \\
Total & - & - & - & $1 \%$ & $1 \%$ & $1 \% \%$ \\
\hline
\end{tabular}

Table 9: Correlation of colposcopy to histopathology

\begin{tabular}{lcccccc}
\hline \multirow{2}{*}{ Colposcopy findings } & \multicolumn{5}{c}{ Histopathology findings } & \multirow{2}{*}{ Total } \\
\cline { 2 - 5 } Normal & Cervicitis & CIN1 & CIN2 & CIN3 & SCC & $56 \%$ \\
Aceto-white areas & $55 \%$ & $1 \%$ & - & - & - & - \\
Fine punctuations & $8 \%$ & $11 \%$ & $2 \%$ & - & - & $1 \%$ \\
Coarse punctuations & $5 \%$ & $4 \%$ & $1 \%$ & $-1 \%$ & - & $10 \%$ \\
Mosaic pattern & $2 \%$ & $6 \%$ & $2 \%$ & - & $1 \%$ & $2 \%$ \\
Total & - & - & $6 \%$ & $1 \%$ & $1 \%$ & $100 \%$ \\
\hline
\end{tabular}


Table 10: Correlation of Pap smear and colposcopy

\begin{tabular}{|c|c|c|c|c|c|c|}
\hline \multirow[b]{2}{*}{ PAP smear findings } & \multicolumn{5}{|c|}{ Histopathology findings } & \multirow[b]{2}{*}{ Total } \\
\hline & Normal & $\begin{array}{c}\text { Fine } \\
\text { punctuations }\end{array}$ & $\begin{array}{c}\text { Aceto-white } \\
\text { areas }\end{array}$ & $\begin{array}{c}\text { Coarse } \\
\text { punctuations }\end{array}$ & $\begin{array}{l}\text { Mosaic } \\
\text { pattern }\end{array}$ & \\
\hline NILM & $56 \%$ & $10 \%$ & $18 \%$ & $3 \%$ & - & $87 \%$ \\
\hline LSIL & - & - & $3 \%$ & $5 \%$ & - & $8 \%$ \\
\hline HSIL & - & $1 \%$ & - & $2 \%$ & $1 \%$ & $4 \%$ \\
\hline Malignancy & - & - & - & - & $1 \%$ & $1 \%$ \\
\hline Total & $56 \%$ & $11 \%$ & $21 \%$ & $10 \%$ & $2 \%$ & $100 \%$ \\
\hline
\end{tabular}

\section{DISCUSSION}

In the present study, Pap smear and colposcopy were done for 100 cases undergoing total hysterectomy. Colposcopy showed a higher sensitivity and specificity than Pap smear in predicting the cervical histopathology. Unexpected abnormal cervical pathology was found in $3.3 \%$ of cases when depending on colposcopy, and in $66.7 \%$ when depending on Pap smear.

Colposcopy as a screening modality has been addressed by many authors, and is superior in accuracy, sensitivity and specificity compared to Pap smear. The study by Sideri et al. showed a good correlation between colposcopic and histopathological findings, with a sensitivity of $90.2 \%$, sensitivity and a specificity of $48.6 \%$ in predicting $\mathrm{CIN}^{[10]}$. In a meta-analysis which included eight longitudinal studies, colposcopic accuracy was $89 \%$ versus histopathology. The sensitivity of colposcopy ranged between 87 and $99 \%$, and specificity ranged between 26 to $87 \% \%^{[11]}$.

Several studies support the incidental finding of abnormal cervical histopathology in hysterectomy specimens for benign indications. Chapman et al. reported that $27 \%$ of women diagnosed with occult cervical cancer at the time of benign hysterectomy had a normal pap smear before their operations ${ }^{[12]}$. In an Indian study, 14 out of 100 women undergoing hysterectomies for benign indications, were later on found to have $\mathrm{CIN}$ in their hysterectomy specimens ${ }^{[13]}$. Another study reviewed supposed benign hysterectomy specimens of 430 patients who all had normal preoperative Pap smears, and found $5(1.2 \%)$ to have abnormal cervical histopathology ${ }^{[14]}$.

\section{Strengths and weaknesses of the study:}

The strength of the study lies in pointing out the importance of cervical screening, and addressing the possibility of having a diseased cervix in patients undergoing hysterectomy. Also, Kasr Al Ainy Hospital of Cairo University in which the study was conducted, is a large university hospital that attracts a wide variation of healthcare seekers from different socioeconomic and educational categories.

Our study is not short of weaknesses. Pap smears at our hospital are carried out in the conventional way, and not by liquid based cytology, and the HPV status of our patients was not known.

\section{CONCLUSION}

Pap smear has a good specificity, but a low sensitivity in predicting cervical histopathology. Meanwhile, colposcopy has a high sensitivity and a reasonable specificity in predicting the histopathology. Colposcopy has a higher sensitivity and specificity as a pathology predictor, when compared to Pap smear. Despite this, unexpected cervical pathology could be detected in hysterectomy specimens postoperatively.

\section{Relevance and possible implications of our findings:}

Colposcopy could be incorporated as a routine cervical screening tool, in combination with Pap smear. Also, in situations where national screening programs are not available, and patients are scheduled for hysterectomy, preoperative cervical assessment by Pap smear and colposcopy is advised to avoid unexpected histopathological findings later. In cases of unknown status of the cervix, it would be reasonable to avoid a subtotal hysterectomy.

\section{CONFLICT OF INTEREST}

There are no conflicts of interest.

\section{REFERENCES}

1. Domingo S, Pellicer A (2009) Overview of current trends in hysterectomy. Expert Rev Obstet Gynecol 4:673-685. https://doi.org/10.1586/eog.09.51.

2. Jones HW (2008) Abdominal Hysterectomy. In: Rock JA, Jones HW (eds) TeLinde's operative 
gynaecology, 10th ed. Lippincott: Williams and Wilkins, Philadelphia, pp 727-743.

3. Schiffman M, Castle PE, Jeronimo J, et al. (2007) Human papillomavirus and cervical cancer. Lancet 370:890-907. https://doi.org/10.1016/ S0140-6736(07)61416-0.

4. Tierney B, Westin SN, Schlumbrecht MP, Ramirez PT (2010) Early cervical neoplasia: advances in screening and treatment modalities. Clin Adv Hematol Oncol 8:547-555.

5. Kohli B, Arya SB, Goel JK, et al. (2014) Comparison of Pap Smear and Colposcopy in Detection of Premalignant Lesions of Cervix. J South Asian Fed Menopause Soc 2:5-8. https://doi.org/10.5005/jp-journals-10032-1023.

6. ACOG Practice Bulletin No. 99: Management of Abnormal Cervical Cytology and Histology (2008) Obstet Gynecol 112:1419-1444. https://doi.org/10.1097/AOG.0b013e318192497c.

7. Mahnert N, Morgan D, Campbell D, et al. (2015) Unexpected Gynecologic Malignancy Diagnosed After Hysterectomy Performed for Benign Indications. Obstet Gynecol 125: https://doi.org/10.1097/AOG.0000000000000642.

8. Hakama M, Chamberlain J, Day NE, et al. (1985) Evaluation of screening programmes for gynaecological cancer. Br J Cancer 52:669-673. https://doi.org/10.1038/bjc.1985.241.

9. Nayar R, Wilbur DC (2015) The Bethesda system for reporting cervical cytology: definitions, criteria, and explanatory notes, 3rd ed. Springer, New York.

10. Sideri M, Spolti N, Spinaci L, et al. (2004) Interobserver variability of colposcopic interpretations and consistency with final histologic results. J Low Genit Tract Dis 8:212-216.

11. Olaniyan OB (2002) Validity of colposcopy in the diagnosis of early cervical neoplasia: a review. Afr J Reprod Health 59-69.

12. Chapman JA, Mannel RS, DiSaia PJ, et al. (1992) Surgical treatment of unexpected invasive cervical cancer found at total hysterectomy. Obstet Gynecol 80:931-934.

13. Visalakshi S, Balakrishnan K, Maheswari KU, Hemalatha G (2016) Incidental Finding of Cervical Dysplasia in Hysterectomy Cases Done for Other Causes. Int J Sci study 4:157-161.

14. Chundarat $P$, Suwannarurk K, Bhamarapravatana $\mathrm{K}$, et al. (2014) Incidental finding of abnormal cervical pathology in hysterectomy specimens after normal preoperative Papanicolaou smears in Thammasat University Hospital. Asian Pacific J Cancer Prev 15:5811-5814. 